\title{
Structural Crystallography of Inorganic Oxysalts
}

SERGEY V. KRIVOVICHEV

Professor and Chairman

Department of Crystallography

St. Petersburg State University 


\section{Contents}

1 Basic concepts

1.1 Structural classification of inorganic oxysalts

1.2 Basic geometrical parameters

1.3 $\mathrm{OH}$ and $\mathrm{H} 20$ in inorganic oxysalts

2 Graph theory applied to low-dimensional structural units in inorganic oxysalts

2.1 Symbolic description of topologies of heteropolyhedral structural units

2.2 2D topologies: graphs with $M-T$ links only

2.2.1 Basic graph \{3.6.3.6 $\}$ and its derivatives 8

2.2.1.1 M:T 1:2 10

2.2.1.2 Topological isomerism 15

2.2.1.3 $M: T=2: 3 \quad 18$

2.2.1.4 $M: T=3: 4 \quad 18$

2.2.1.5 $M: T=13: 18,3: 8,5: 8,3: 5,1: 1$ and $1: 3$

2.2.1.6 Geometrical isomerism and orientation matrix 24

2.2.1.7 Cis-trans geometrical isomerism and connectivity diagrams $\quad 34$

2.2.2 Autunite topology and its derivatives 35

2.2.3 Other basic graphs and their derivatives $\quad 40$

2.2.4 Modular approach to complex 2D topologies 42

2.2.4.1 Topologies derived from the same basic graph 42

2.2.4.2 Topologies derived from more than one basic graph 46

$2.3 \quad$ 2D topologies: graphs with $M=1$ links and without $M-M$ links 46

$2.42 \mathrm{D}$ topologies: graphs with $M-M, M=M$, or $M-=M$ links 52

2.4.1 Graphs with $M-M$ links $\quad 52$

2.4.1.1 Graphs with finite subgraphs of black vertices 54

2.4.1.2 Graphs with 1D subgraphs of black vertices $\quad 54$

2.4.1.3 Graphs with 2D subgraphs of black vertices 59

2.4.1.4 Graphs with modular structure 60

2.4.1.5 Other graphs 64

2.4.2 Graphs with $M=M$ links $\quad 65$

2.4.3 Graphs with $M$-=-M links 68 
2.5 1D topologies: chains 69

2.5.1 Some notes 69

2.5.2 Orientational geometrical isomerism of chains $\quad 72$

2.5.3 Lone-electron-pair-induced geometrical isomerism $\quad 77$

2.5.4 Cis—trans isomerism $\quad 79$

2.6 OD topologies: finite clusters 81

2.7 Nanoscale low-dimensional units in inorganic
oxysalts: some examples

3 Topology of framework structures in inorganic oxysalts 94

3.1 Regular and quasiregular nets 94

3.2 Heteropolyhedral frameworks: classification principles 96

3.3 Frameworks based upon fundamental building blocks (FBBs) 98

$\begin{array}{lll}\text { 3.3.1 Some definitions } & 98\end{array}$

3.3.2 Leucophosphite-type frameworks 98

3.3.3 Frameworks with oxocentered tetrahedral cores $\quad 100$

3.3.4 Pharmacosiderite-related frameworks 102

3.3.5 Nasicon, langbeinite and related frameworks 103

3.4 Frameworks based upon polyhedral units 105

$\begin{array}{lll}3.4 .1 & \text { Polyhedra } & 106\end{array}$

3.4.2 Tilings 106

3.4.3 Example 1: minerals of the labuntsovite group 107

3.4.4 Example 2: shcherbakovite—batisite series 108

3.4.5 Combinatorial topology of polyhedral units 109

3.4.6 Topological complexity of polyhedral units: petarasite net

3.5 Frameworks based upon infinite chains

3.5.1 Fundamental chains as bases of complex frameworks: an example

3.5.2 Frameworks with non-parallel orientations of fundamental chains

3.5.3 Frameworks with no $M-M$ and $T-T$ linkages

3.5.3.1 Frameworks consisting of kroehnkite chains 115

3.5.3.2 Other examples

3.5.4 Frameworks with $M-M$ and no $T-T$ linkages

3.5.4.1 Frameworks based upon chains of corner-sharing $M$ octahedra

3.5.4.2 Frameworks consisting of finite clusters of corner-sharing octahedra

3.5.5 Frameworks with $M=M$ and no T-T linkages 129

3.5.6 Some frameworks based upon T207 double tetrahedra 131

3.5.7 Frameworks with both $T-T$ and $M-M$ linkages 134

3.5.7.1 Zorite and ETS-4 134

3.5.7.2 Benitoite net as based upon arrangement of polyhedral units and tubular units 
3.5.7.3 Tubular units, their topology, symmetry and classification

3.6 Frameworks based upon 2D units

3.6.1 Frameworks based upon sheets with no $T-T$ linkages $\quad 140$

3.6.2 Frameworks based upon sheets with $T-T$ linkages $\quad 143$

3.6.2.1 Octahedral—-tetrahedral frameworks with 2D tetrahedral anions

3.6.2.2 Umbite-related frameworks

3.6.2.3 The use of $2 \mathrm{D}$ nets to recognize structural relationships

3.6.3 Structure description versus intuition

4 Anion-topology approach

4.1 The concept of anion topology 163

4.2 Classification of anion topologies 164

$\begin{array}{ll}4.3 & \text { Anion topologies and isomerism } \\ & 169\end{array}$

4.4 1D derivatives of anion topologies 171

5 Alternative approaches to structure description 173

5.1 Introductory remarks 173

5.2 Anion-centered tetrahedra in inorganic oxysalts 173

5.2.1 Historical notes 173

5.2.2 OD units of oxocentered tetrahedra 175

5.2.3 1D units of oxocentered tetrahedra 179

5.2.4 2D units of anion-centered tetrahedra 187

5.2.5 3D units of anion-centered tetrahedra 198

5.2.6 Which cations may form anion-centered tetrahedra? 201

5.3 Anion-centered octahedra in inorganic oxysalts 203

5.3.1 Some notes on antiperovskites 203

5.3.2 Chemical considerations 204

5.3.3 Structural diversity 204

5.4 Cation arrays in inorganic oxysalts 209

6 Dimensional reduction in inorganic oxysalts 215

6.1 Introduction 215

6.2 Alkali-metal uranyl molybdates 215

6.3 Inorganic oxysalts in the Ca3(T04)2 - 1-13(T04) - (H20) system 217

6.4 Inorganic oxysalts in the M2(T04)3 - H2(T04) - (H20) system 221

6.5 Inorganic oxysalts in the M(T04) - H2(T04) - (H20) system 224

6.6 Concluding remarks 225

References 227

Index 305 